\title{
Similarity of auditory stimuli and generalization of skin conductance response habituation
}

\author{
GERSHON BEN-SHAKHAR and ISRAEL LIEBLICH \\ Hebrew University, Jerusalem, Israel
}

\begin{abstract}
Two alternative models for orienting response habituation to complex sequences of stimuli were formulated. Model 1 assumed complete generalization of habituation, while Model 2 assumed independent habituation processes for the different stimuli making up the complex sequence. Two experiments were conducted in order to compare the models. Experiment 1 was conducted to obtain a simple habituation function to tones. Experiment 2 utilized complex sequences, each comprising two tones. The degree of similarity between these two tones was manipulated by systematically varying the frequency of one of these tones, using a betweensubjects design. The predictions of the two models were correlated with the SCRs of each subject. The results showed that the validities of the models depend on the similarity between the stimuli making up the complex sequence. For a sequence comprising very similar stimuli, Model 1 is a better description of the habituation process. Model 2, however, yields a closer fit to the habituation process to sequences comprising clearly discriminable stimuli.
\end{abstract}

The nature of orienting response (OR) habituation to complex sequences of stimuli is of theoretical importance for the understanding of neuronal models. Several studies have tried to clarify the habituation pattern in such sequences (e.g., Furedy, 1968; Zimny, Pawlick, \& Saur, 1969). Connolly and Frith (1978a, 1978b) have raised the possibility that different laws may govern the habituation process in complex and simple sequences of stimuli. These authors have suggested that the neuronal model operates within a range of specific limits. It follows that if the stimuli making up the complex sequence vary within these limits, the habituation pattern should not differ from that of habituation to a simple sequence. Only when the stimuli set includes a stimulus that falls outside these limits should we expect habituation pattern to the complex sequence that is different from that of habituation to a simple sequence.

Ben-Shakhar (1980) has also dealt with habituation processes to complex sequences, and has tried to formulate, by means of simple mathematical models, the relationship between habituation in complex and simple sequences. Two main models have been formulated: Model 1, which assumes complete generalization of habituation, states that the response in a given trial of a complex sequence is a function of all the previous stimuli in that sequence. In more formal terms, Model 1 states: $g(i / j)=f(i+j)$, where $f$ denotes

The present study was supported by a grant from the Eshkol Institute, Faculty of Social Sciences, The Hebrew University of Jerusalem. We wish to thank Ms. Judy Lustig and Ms. Aya Poznanski for their assistance. the habituation function in a simple sequence, $g$ denotes the habituation function in a complex sequence of stimuli, $i$ and $j$ denote the trial numbers of two stimuli, and $g(i / j)$ denotes the response to a stimulus in its $i^{\text {th }}$ presentation, given that the other stimuli have appeared $j$ times before it. Model 2 assumes that the response to a given stimulus is determined solely by the number of previous presentations of that stimulus only. In more formal terms, Model 2 states: $g(i / j)=f(i)$. Model 2 assumes that generalization of habituation does not take place in some complex sequences. In terms of Sokolov's (1963) "neuronal model" theory, it could be said that Model 2 assumes a separate neuronal model for each stimulus, while Model 1 assumes a single neuronal model for the whole series. In terms of Connolly and Frith (1978a, 1978b), it can be stated that Model 1 describes a situation in which all the stimuli making up the complex sequence fall within the range of operation of the neuronal model, while Model 2 describes a situation in which at least one stimulus falls outside this range. It should be emphasized that all the models suggested by Ben-Shakhar (1980) and by Ben-Shakhar, Dymshitz, and Lieblich (1982) fall within the general theoretical framework of Sokolov (1963) and do not constitute a new theory of the habituation process. They relate to different possible habituation patterns in complex sequences, and these patterns depend mainly on the specific stimuli making up a particular sequence. Previous studies have indicated that Model 2 is a better description of SCR habituation processes to complex sequences than is Model 1. All of these studies used meaningful verbal stimuli such as numbers, names, etc., some of which were made relevant to the subjects through 
certain instructions. The empirical generalization stated above could be limited by two factors: (1) the fact that all stimuli can be verbally encoded, and (2) the fact that, by the instructions, some stimuli can be made strongly salient relative to others. The present study attempts to test the models in a more general setup in which simple auditory stimuli are presented without any relevance imposed on the stimuli by instructions. The use of simple auditory stimuli which vary continuously made it possible to investigate the effects of stimulus similarity on generalization of habituation in complex sequences. As hypothesized previously (Ben-Shakhar, 1980), the more similar the stimuli making up the complex sequence, the more likely it is that Model 1 will yield a better fit of the complex habituation process than will Model 2, since the likelihood that a single neuronal model will develop for a whole set of stimuli is positively related to the similarity of the stimuli within this set. In order to compare the two models, two experiments were conducted. The aim of Experiment 1 was to derive the simple habituation function for tones. Experiment 2 focused on the comparisons of the predictions derived from the two models with regard to the habituation process in complex sequences. The predictions were based on the simple function derived from Experiment 1.

\section{EXPERIMENT 1}

\section{Method}

Subjects. One hundred and seven female and 93 male Hebrew University undergraduate students participated in the experiment. They were recruited through an advertisement and were paid for their participation.

Apparatus. A constant-current $(18 \mu \mathrm{A})$ skin resistance response (SRR) apparatus was direct coupled to a Brush 2 recorder. The recording system was placed in a control room separated by a oneway mirror from the subject's room. Beckman electrodes were used for skin resistance measurement. The current density was $31.75 \mu \mathrm{A} / \mathrm{cm}^{2}$. A PDP-8/E computer was used to transform the SRR into SCR. The same computer was used to control presentation of the tones. A Wavetek Model 164 wave generator was used to generate the pure sine-wave tones which were presented through AKG Model K60 earphones.

Procedure. The subject was seated at a table facing a blank wall. Electrodes were attached to the volar side of the index and the fourth fingers of the subject's left hand, using masking tape with pressure such that the subject felt comfortable. Beckman electrode paste, which contained, according to the manufacturer's label, sodium chloride, glycerin, tragacanth, and benzyl alcohol .5\%, was used to ensure proper contact. All testing was carried out in an air-conditioned laboratory maintained at $26^{\circ} \mathrm{C}$. The subjects were instructed to sit quietly and not move. The earphones were then placed on the subject's ears. At the end of $2 \mathrm{~min}$ baseline recording, the subject was told that he/she would hear a sequence of tones. $\mathrm{He} / \mathrm{she}$ was instructed to listen to the tones quietly and to pay attention to possible differences between the tones. Five sequences of tones were used. All tones were presented at $70 \mathrm{~dB}$ at the level of the subject's ear. The following tones were used: $A$, $1,000 \mathrm{~Hz} ; \mathrm{B}, 1,004 \mathrm{~Hz}$; C, 1,016 Hz; D, $1,064 \mathrm{~Hz}$; and E, $1,256 \mathrm{~Hz}$. Each sequence comprised 16 repetitions of one of the above tones. The interstimulus intervals were determined randomly within the range of 11-19 $\mathrm{sec}$ with a mean of $15 \mathrm{sec}$. Tone duration was $1 \mathrm{sec}$.
The following rotations of the sequences were used: A,B,C,D,E; B,C,D,E,A; C,D,E,A,B; D,E,A,B,C; E,A,B,C,D. Note that A stands for a sequence of 16 repetitions of stimulus $A$. The subjects were divided randomly into five equal groups of 40 subjects each. Each group was assigned to one of the five rotations. Following each sequence, the earphones were removed from the subjects for a 1-min rest.

\section{Results}

Analysis of the response to each tone was carried out by determining the maximal conductance change observed within $5 \mathrm{sec}$ after the offset of the tone. To eliminate individual differences in reactivity, the conductance changes to all 80 tones were transformed into standard scores relative to each subject's mean and standard deviation. An empirical habituation curve was constructed for each tone (A to $\mathrm{E}$ ) by averaging the standard scores from each of the 16 trials across all subjects. (The resulting means were based on responses to the same tone, pooled across the different sequences.) Following Ben-Shakhar, Lieblich, and Kugelmass (1975), negative exponential functions were fitted to the five empirical curves. The fitted functions were of the following general form: $f(i)=a e^{-\lambda(i-1)}+b$, where $i=1 \ldots 16$. Five additional empirical curves were constructed for the first through fifth sequence by averaging the standard scores from each of the 16 trials in each sequence across all subjects. (The resulting means were based on responses to different tones for the different groups.) Again, negative exponential functions were fitted to these five empirical curves. There were no differences between the functions fitted to the different tones with regard to any of the parameters. There were, however, differences in the functions fitted to the different sequences. As a result, it was decided to use the data of only the first sequence pooled across the different tones.

The assumption underlying the sequences rotation design is that the habituation pattern could be determined only by the stimulus type (defined here by the frequencies of the tones) and by the order position of the stimulus sequence, without carry-over effect from the specific type of sequence that had been presented previously. This may be important, since, in the present design, each sequence always followed the same prior sequence (unless it was the first sequence presented to the subject). If the assumption described did not hold, then our comparisons might be affected. Note, however, that this should not influence the comparisons of the models to be used in Experiment 2, since only the first of each subject's sequences was used to construct the habituation function. The following function resulted in the best fit, with the empirical curve based on the 16 averages of the first sequence: $f(i)=1.36, e^{-1.04(i-1)}-.16$. The Pearson correlation coefficient between the observed means and the corresponding values of the fitted function was .94. 


\section{EXPERIMENT 2}

The purpose of Experiment 2 was to compare the two models described above, using complex sequences of tones. Each sequence comprised two tones: a standard tone of $1,000 \mathrm{~Hz}$ and one of the other four tones used in Experiment 1. The proportions of the two tones were manipulated. It was hypothesized that the more dissimilar the two tones were, the better would Model 2 describe the habituation process in the complex sequences.

\section{Method}

Subjects. One hundred and three female and 100 male Hebrew University undergraduate students participated in the experiment. They were recruited through an advertisement and were paid for their participation.

Apparatus. The apparatus was the same as that used in Experiment 1.

Procedure. The procedure was the same as that of Experiment 1 except for the following: Only one sequence of 16 trials was presented to each subject. As stated above, each sequence comprised two stimuli: standard stimulus $A$ and $B, C, D$, or E. Five mixtures of the two tones were constructed for each of the four pairs (A,B, $A, C, A, D$, and A,E). These five mixtures differed in the proportions of the two tones. The proportions of the standard tone, $A$, were $1 / 8,1 / 4,1 / 2,3 / 4$, and $7 / 8$. The specific sequence of 16 stimuli was determined randomly for each proportion group, resulting in five sequences. For example, using tone $B$, the following sequences would be obtained: (1) B B B B B A B B A B B B B B B B, (2) B B B A B B B B A B A B B A B B, (3) A B B A B A B B B B A A A A B , (4) B A A A A B A A B A A A B , and (5) A A A A A A A B A A A A A B A. Tones C, D, and E replace tone $B$ in identical patterns. The four pairs of tones and the five mixtures resulted in 20 experimental groups. The subjects were assigned randomly to those 20 groups under the constraint of at least 10 subjects per group.

At the end of the experiment, 84 of the subjects were tested for discrimination between the tones. They were presented with the four pairs of tones used in the experiment (pairs A,B, A,C, $A, D$, and $A, E)$. Each pair was presented twice in random order. Each tone in a pair was presented for $1 \mathrm{sec}$ with a 1-sec interval between the two tones within a pair. The order of the tones within each pair was reversed in its second presentation. The subjects were instructed to estimate the similarity of the two tones in each pair on an 8-point scale, with $1=$ tones are identical and $8=$ tones are extremely different. The subjects were instructed to make their judgments immediately after the presentation of each pair.

\section{Results}

The measurement and standardization of the responses were carried out as in Experiment 1. The expected standard score of each trial was computed using each of the two models described in the introduction. These expected values were based on the $f(i)$ derived from Experiment 1 . Since the predictions of the two models are based on the same simple habituation function, they are not independent; in fact, their predictions are highly correlated. It was therefore decided to use partial correlations of the predictions of each model with the observed data, holding constant the predictions of the other model. This procedure, it was felt, might sharpen the distinctions between the predictions of the models and thus provide a better means for comparison. For each subject, two partial Pearson correlation coefficients were computed: (1) the partial correlation between standardized observed responses and the corresponding expected values predicted by Model 1, partialing out the predictions of Model 2, and (2) the partial correlation between the observed data and the results expected by Model 2, partialing out the predictions of Model 1. All these correlations were computed across 16 trials within each subject.

The goal of the present analysis was to compare the two distributions of partial correlations obtained by the two models. Since correlation coefficients (and partial correlations) are not normally distributed, it was necessary to transform them into Fisher's $\mathrm{Z}$ scores, in order to meet the normality assumption underlying the statistical tests to be conducted. Thus, each correlation was transformed into a Fisher $\mathbf{Z}$ value. Differences between the $Z$ values obtained from the correlations of Model 1 with the data and the $Z$ values obtained from the correlations of Model 2 with the data were computed. Table 1 presents the means of these differences for each experimental condition. Since the probability conditions of $1 / 8$ and $7 / 8$ as well as $1 / 4$ and $3 / 4$ are equivalent, they were combined in the analysis.

The results of the postexperimental discrimination test showed that tones $A, B$ and tones $A, C$ were perceived as almost identical, while tones $A, D$ and $A, E$ were effectively discriminated. The median scores on the 8-point scale across the 84 subjects were: $1,2.5$, 5.5, and 7.5 for pairs $A, B, A, C, A, D$, and $A, E$, respectively. The proportions of subjects who produced scores of at least 5 were: $0, .14, .69$, and .94 for pairs $A, B, A, C, A, D$, and $A, E$, respectively. On the basis of these results, it was decided to group A,B with $A, C$ and $A, D$ with A,E. A $3 \times 2$ ANOVA was performed on Fisher's Z difference scores. A significant stimulus similarity effect was obtained $[F(1,197)$ $=5.04, \mathrm{MSe}=1.23]$. No significant interaction and no probability effects were obtained $[F(2,197)=.84$ and $F(2,197)=.83$ for these two effects, respectively]. No significant differences were obtained when Fisher's $Z$ difference scores of the A,B groups were compared with those of the $A, C$ group or when the $Z$ differences

Table 1

Mean Differences of Fisher Z Transformation Scores for Three Stimulus Probability Conditions Crossed With Four Stimuli Pairs

\begin{tabular}{lllrr}
\hline & \multicolumn{4}{c}{ Ptimuli Pair } \\
\cline { 2 - 5 } \multicolumn{1}{c}{ Condition } & A,B & A,C & A,D & \multicolumn{1}{c}{ A,E } \\
\hline $7 / 8$ and $1 / 8$ & .364 & .003 & -.160 & .004 \\
$3 / 4$ and $1 / 4$ & .185 & .270 & .129 & .065 \\
$1 / 2$ & .099 & .086 & -.295 & -.337 \\
Mean Differences & .216 & .130 & -.109 & -.089 \\
\hline
\end{tabular}

Note-The differences were always defined as the $Z$ score produced by partial correlations of Model 1 minus the $Z$ score produced by partial correlations of Model 2. 
of the A,D group were compared with those of the A,E group.

Essentially the same results were obtained when other equivalent analyses were performed, and when different statistics were used, that is, correlations between the predictions of the models and the mean standardized responses of each experimental condition.

\section{DISCUSSION}

The main result of Experiment 2 was that the predictive power of the two models is dependent upon the psychological distance between the stimuli making up the complex sequence.

In a situation in which the psychological distance between the stimuli is small (in terms of the subject's ability to discriminate between them), Model 1, which assumes complete generalization of habituation, provides a better fit than does Model 2. On the other hand, Model 2, which assumes independent habituation processes to the different stimuli of the complex sequence, describes better the data in a situation in which the psychological distance between the stimuli is relatively large. This result is in line with the concept of dichotomization suggested previously by the authors (Ben-Shakhar, 1977; Ben-Shakhar et al., 1975; Lieblich, Kugelmass, \& Ben-Shakhar, 1970). It is logical to suggest that, in a complex sequence in which the different stimuli are for some reason indiscriminable, the sequence will be processed as a single category. In such a case, one might expect that a single neuronal model will be constructed for all stimuli, resulting in habituation described by Model 1. On the other hand, when stimuli of the complex sequence are discriminable, the stimuli are classified into distinct categories. For each category, a neuronal model is constructed, and thus the habituation process is described by Model 2. The present result is in line with the conclusion of Connolly and Frith (1978a, 1978b) that the neuronal model operates within a range of specific limits. It is possible that those limits accord with the subjective judgments of the subjects; thus, there may be a correspondence between similarity judgments and the habituation pattern. The present study compared two models which describe two extreme possibilities for the complex habituation pattern. Ben-Shakhar (1980) suggested that a combination of Models 1 and 2 could be a more proper description of the habituation process in complex sequences than either of the extreme cases. The combined model could be formulated as follows: $g(i / j)=S(A, B) \cdot f(i+j)+[1-S(A, B)]$ $f(i)$, where $S(A, B)$ is a parameter for the degree of similarity between the stimuli (A and B) making up the complex sequence. This parameter assumes values between 0.0 (for extremely different stimuli) and 1.0 (for identical, i.e., extremely similar stimuli). Note that Models 1 and 2 are special cases of this combined model. In order to apply this model, one must estimate the similarity parameters. Two approaches present themselves: (1) the parameter $\mathrm{S}(\mathrm{A}, \mathrm{B})$ is a continuous function of the perceived similarity between $A$ and $B$; (2) the parameter $S(A, B)$ equals 1.0 only when the perceived similarity exceeds a certain threshold value (up to this threshold, $S=0$ ). We tried to examine these two possibilities using the present results. The values of the continuous Ss derived from the results of the postexperimental discrimination test were: $0, .14, .69$, and .94 for pairs A,B, A,C, A,D, and A,E, respectively. The values of the Ss for the threshold approach were: $0,0,1.0$, and 1.0 for the above four conditions, respectively. Unfortunately, it was impossible to distinguish between the predictions of these two combination models, since they were very highly correlated (for most subjects, the correlation between the predictions of the two models was at least .96). It is worth noting that the results of the present experiment indicated that there was no increase in the advantage of Model 1 over Model 2 when moving from an A,B sequence to an $A, C$ sequence, and no increase in the advantage of Model 2 over Model 1 when moving from an A,D to an $A, E$ sequence. These facts may indicate that the threshold possibility is more plausible than the continuous one. However, this conclusion must be considered with great caution, since the present results may depend on the specific choices of the tones. For instance, it is possible that inclusion of additional sequences of tones with differences ranging between 16 and $64 \mathrm{~Hz}$ would have changed the results such that the usefulness of Model 1 would gradually decrease as the difference approached $64 \mathrm{~Hz}$. This question requires further collection of data. The advantage of the combined model is in its generality. If we accept this formulation, it means that habituation processes to complex and simple sequences are governed by the same laws and that the only difference between them is in the parameter S. This possibility is in contrast with the conclusions drawn by Connolly and Frith (1978a, 1978b).

It should be noted that, unlike previous investigators (Ben-Shakhar, 1977; Ben-Shakhar et al., 1975; Ben-Shakhar, Lieblich, \& Kugelmass, 1982; Lieblich et al., 1970), we did not find that the stimulus probability factor had an influence on the relative validities of the two models. It should be emphasized, however, that, unlike the previous studies, which attached relevance to some of the stimuli, the present stimuli were all neutral. In this regard, the present results may be seen as a generalization of previous studies in which variants of Models 1 and 2 were tested in a situation in which the discrimination between the stimuli was perfect, on 
the basis of relevance to the subject. We can conclude that relevance is not a necessary condition for Model 2, although it may be a sufficient condition. Thus, the present results are a generalization of the applicability of Model 2 to a wider set of complex sequences.

\section{REFERENCES}

Ben-Shakhar, G. A further study of the dichotomization theory in detection of information. Psychophysiology, 1977, 14, 408-413.

Ben-Shakhar, G. Habituation of the orienting response to complex sequences of stimuli. Psychophysiology, 1980, 17, 524-534.

Ben-Shakhar, G., Dymshitz, J., \& Lieblich, I. Generalization of habituation of skin conductance response (SCR) to multidimensional sequences of stimuli. Psychophysiology, 1982, 19, 178-182.

Ben-Shakhar, G., Lieblich, I., \& Kugelmass, S. Detection of information and GSR habituation: An attempt to derive detection efficiency from two habituation curves. Psychophysiology, 1975, 12, 283-288.

Ben-Shakhar, G., Lieblich, I., \& Kugelmass, S. Interactive effects of stimulus probability and significance on the skin conductance response. Psychophysiology, 1982, 19, 112-114.
Connolly, J. F., \& Frith, C. D. Effects of stimulus variability on the amplitude and habituation of the electrodermal orienting response. Psychophysiology, 1978, 15, 550-555. (a)

Connolly, J. F., \& Frith, C. D. Effects of varying stimulus context on habituation and sensitization of the O.R. Physiology \& Behavior, 1978, 21, 511-514. (b)

FUREDY, J. Human orienting reaction as a function of electrodermal versus plethysmographic response modes and single versus alternating stimulus series. Journal of Experimental Psychology, 1968, 77, 70-78.

Lieblich, I., Kugelmass, S., \& Ben-Shakhar, G. Efficiency of GSR detection of information as a function of stimulus set size. Psychophysiology, 1970, 6, 601-608.

Sokolov, E. Perception and the conditioned reflex. New York: Pergamon Press, 1963.

Zimny, G. H., PAwlick, G. F., \& SAUR, D. P. Effects of stimulus order and novelty on orienting responses. Psychophysiology, $1969,5,166-173$.

\section{NOTE}

1. A rejection region of $p<.05$ was used in all statistical tests.

(Manuscript received August 31, 1981; revision accepted for publication February 3, 1982.) 